\title{
Influence of Electrical Joint Compound on Electrical Contact Overheating of Overhead Line
}

\author{
Wang Guogang ${ }^{1, a}$, Zhao Yueju ${ }^{1, b}$, Wu Liusuo ${ }^{1, c}$, Kong Zhigang $^{2, d}$ \\ ${ }^{1}$ Beijing Guodian Futong Science and Technology Development Co.,Ltd., Beijing 100070, China; \\ ${ }^{2}$ Beijing University of Posts and Communications, Beijing, 100876, China \\ assnabi@126.com, bzhaoyj@iccas.ac.cn, cwuliusuo @sgepri.sgcc.com.cn, dzgkong@bupt.edu.cn
}

Keywords: electrical joint compound; electrical connection; contact resistance

\begin{abstract}
Influence of electrical joint compound on electrical contact overheating energy consumption is studied in this paper. The high current test were made under different current(400A 、600A 、800A and 1000A). Aluminum-aluminum connections are selected to conduct contact resistance test and temperature rise test. The results show that contact resistance is reduced about $50 \%$, and the temperature of electrical connection decreased $20^{\circ} \mathrm{C}$. The results show that there is remarkable effect after applying the compound. Electrical joint compound can reduce overheating defects and increase the reliability of electrical connection.
\end{abstract}

\section{Introduction}

Overheating of electrical contact is a very common phenomenon during the service of transmission and transformation equipment. It occurs at the connection joints of network [1-3] and super high voltage equipment and super current lines [4].

The overheating defects are always a serious problem in electric network. The overheating of electrical contact not only increases wastage, but also causes a transformation accident. With the increase of running load, the overheating defects of electrical contact become more frequency and serious.

Many researchers studied the formation of the overheating defects, electrical contact materials, corrosion mode, equipment degradation, incorrect operation, service environmental, et al [5-7]. Moreover, some researchers proposed the prevention methods to reduce overheating, which are useful in the safety of network [8]. However, they only focused on practical phenomenon and lacking the analysis of mechanisms. In this paper we investigated the application of electrical joint compound, which demonstrated to be one of the most effective materials to solve overheating problem.

\section{Experimental materials and methods}

Wire clip is chosen according to the standard requirement and the size is $100 \mathrm{~mm} \times 60 \mathrm{~mm}$. Fig. 1 shows the sketch of tested samples. Punch two holes in the top of aluminum wire clip and fix every two board together with bolt. According to the samples were painted with electrical joint compound or without, the samples are classified into two categories: A: Polish the contact surface and clean with alcohol, then put them together and tighten up with nut. B: Polish the contact surface and clean with alcohol, evenly paint the surface with $0.2 \mathrm{~mm}$-thick electrical joint compound; put the surface together and tighten up with nut. 


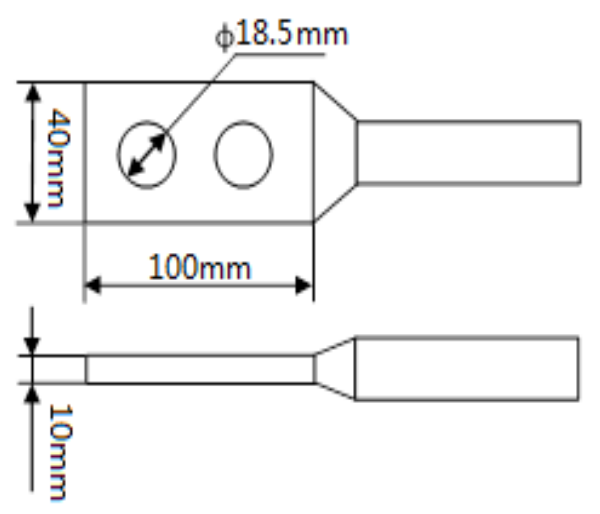

Fig. 1 Sketch of tested samples.

The experimental electric current is set at 400A, 600A, 800A, 1000A, respectively. Two holes in the top of aluminum wire clip were used to fix two boards together with bolt. The contact resistance is measured using digital micro-ohmmeter, and the schematic of measure method is showed in Fig. 2.

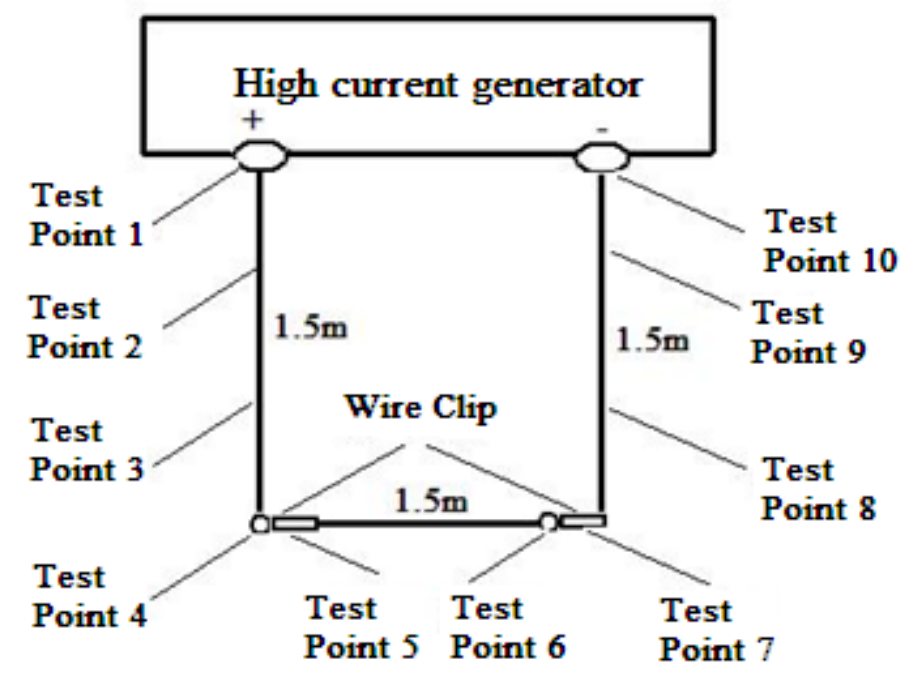

Fig.2 Schematic of high current test

\section{Experimental results and discussion}

The temperature rise rate of electrical contact can directly indicate the heat loss and electric energy loss. This experiment found the relationship between temperature of electrical contact and working time, as shown in Fig. 3 and Fig. 4. The highest temperatures of electrical contact with and without electrical compound are shown in the Fig. 5.

It can be seen from the Fig. 3 and Fig. 4, the temperature increase with the time, while the higer the current is, the higer the temperature rise rate is. For example, for the samples of $\mathrm{A}$, the temperature of electrical contact rise slowly during 20 minutes from $21.0^{\circ} \mathrm{C}$ to $22.8^{\circ} \mathrm{C}$ under the condition of $400 \mathrm{~A}$, while the temperature rises rapidly during 20 minutes from $21.0^{\circ} \mathrm{C}$ to $64.9^{\circ} \mathrm{C}$ under the condition of 1000A. With further extension of time, the temperature of $\mathrm{A}$ and $\mathrm{B}$ samples arrive at $40.2^{\circ} \mathrm{C}$ and $230.5^{\circ} \mathrm{C}$ by the end of the test. After test, the color of localized pitting area becomes dark. However, there is no significant change in contact surface of sample. For the samples $\mathrm{B}$, it can be seen that the temperature is about $44^{\circ} \mathrm{C}$ under the condition of $400 \mathrm{~A}$, which is similar to that of samples A. As for the $1000 \mathrm{~A}$, the temperature is 210 than that containing electrical joint compound, as shown in Fig.5. It means electrical joint 
compound can effectively decrease the temperature of electrical contact and reduce the overheating defects.

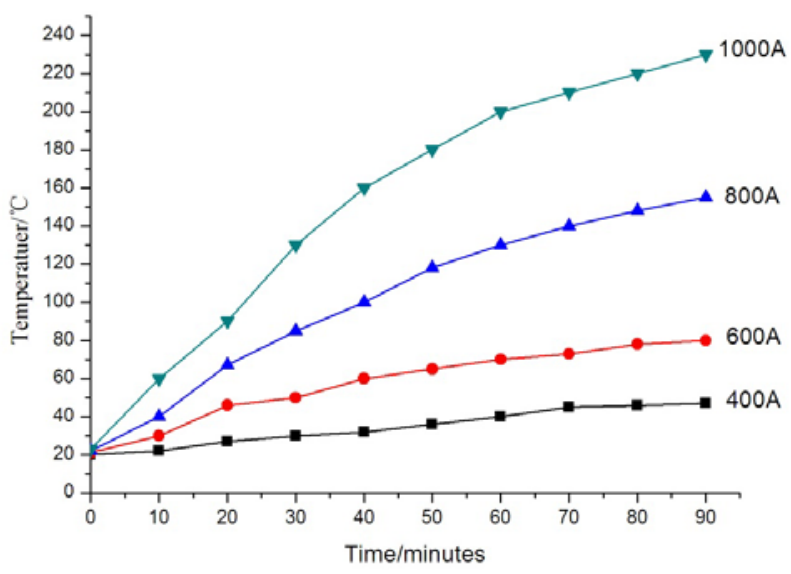

Fig.3 The relationship between current and temperature without electrical joint compound (Samples A)

The results show that the temperature of electrical connection decreased $20^{\circ} \mathrm{C}$, thus there is remarkable energy conservation after applying the compound. Electrical joint compound can fill the gap between two metal contact surfaces. These conducting particles in compound electrically join through "contact effect" and mutually contact under impressed pressure. "Contact effect" is the function of the electrical resistivity of conducting particles. The lower of electrical resistivity is, the higher of electrical conductivity is [9-10]. Besides the "contact effect", electrical joint compound also has "tunneling effect". Some conducting particles are not mutually contacted, but are very close to each other. These particles can conduct by electron transition and form tunnel current by thermal vibration [11-12].

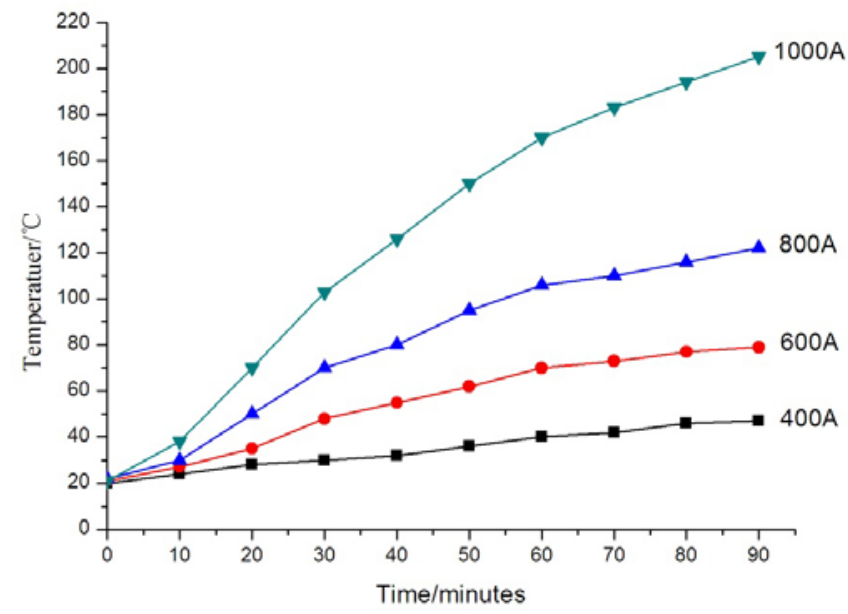

Fig.4 The relationship between current and temperature with electrical joint compound(Samples B) 


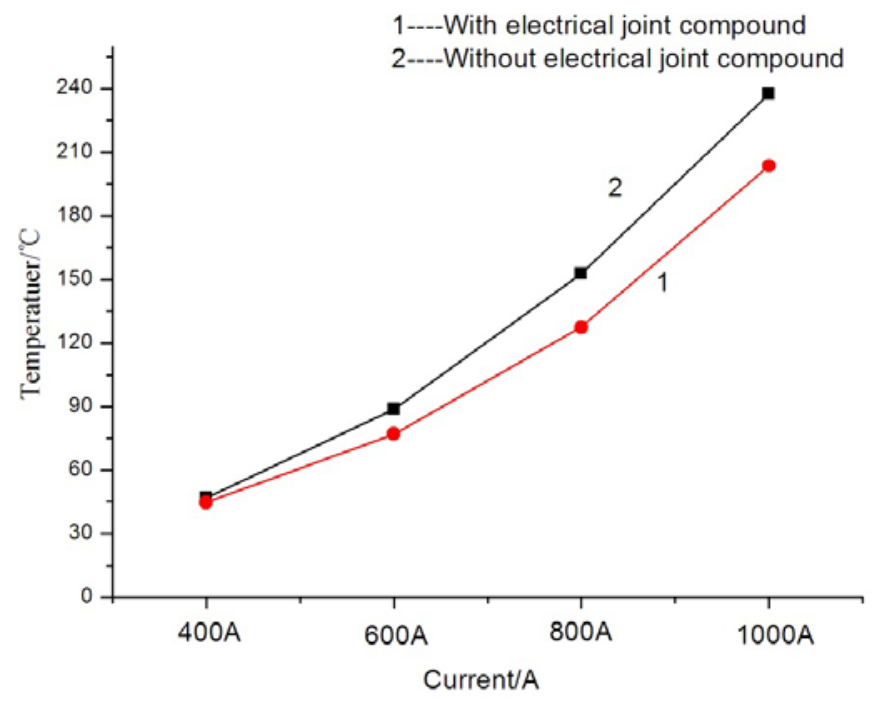

Fig. 5 The variation of the highest temperature on electrical contact versus time

The electrical resistances are tested by the four point method. After polishing wire clip contact surface to roughness $600 \#$ and cleaning with alcohol or acetone, electrical joint compound $(0.2$ mm-thick) is painted on the contact surface of wire clip. The contact resistances with and without electrical joint compound are shown in Fig.6. It can be seen that although the contact resistance of all samples increased after high current test, the contact resistance of B samples is smaller than that of A samples. It indicates that electrical joint compound can reduce the electrical resistance of electrical contact obviously.

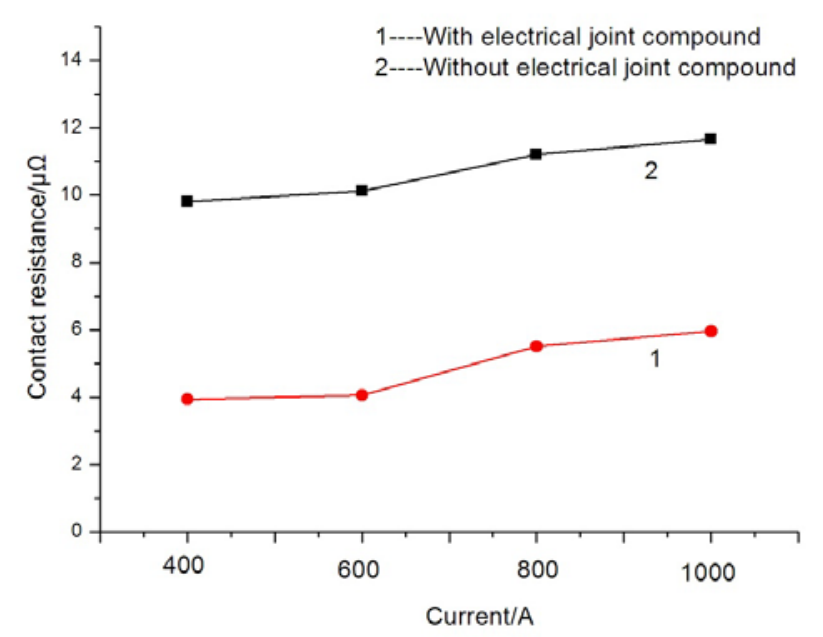

Fig. 6.The contact resistances with and without electrical joint compound

The experiment results show that the electrical joint compound has obvious effect on the contact resistance, so it can effects the economic benefits of power network. For example, the electrical resistance of disconnecting switch can be reduced about 35\% with electrical joint compound. If the contact area of connecting switch is $100 \mathrm{mmx} 60 \mathrm{~mm}$, the current is $500 \mathrm{~A}$, and the operate time is $6000 \mathrm{~h}$ per year. So the amount of electricity saved is:

$$
\Delta Q=I^{2} \Delta R t=500^{2} \times(12.53-7.027) \times 10^{-6} \times 6000=8.25 K W h
$$

Acording to the statistics results, there are about 1000 switches in the power station,so the amount of the electricity saved is about $8000 \mathrm{KWh}$. If the price of electricity is $0.6 \mathrm{yuan} / \mathrm{KWh}$, the cost can be reduced 4800 yuan 。

In addtion, it is found that electrical joint compound can improve corrosion resisting property. The reason is that electrical joint compound can fill in the gap between the two contact surfaces, the corrosive substance cannot penetrate into the gap and corrode metals. So the electrical joint 
compound extend the lifetime of connector, which reduce the costs of replacement of equipment and artificial maintenance.

\section{Summary}

Influence of electrical joint compound on electrical contact overheating energy consumption is studied. The contact resistances were measured by four-point method, and the main conclusions are as follows:

1) Electrical joint compound can effectively decrease the overheating defects of electrical contact. The electrical joint compound can decrease contact resistance by $50 \%$ and lower temperature by $20^{\circ} \mathrm{C}$.

2) Electrical joint compound can decrease the contact resistance of electrical contact and reduce the overheating defects because of "contact effect", "tunneling effect" and "shielding effect".

3) The experiment results show that the electrical joint compound has obvious effect on the contact resistance, so it can effects the economic benefits of power network.

\section{Acknowledgments}

The financial support of this work extended by the Science and Technology Project of State Grid Electric Power Company (No.FTZX201404, WBS : 52467o140004).

\section{References}

[1] J. Jing: High Voltage Electrical Apparatus, vol. 08(2012), p. 114

[2] X.W.Li, H.Lv and Q.F.Lu: Guangdong Electric Power, Vol. 09(2012), P.103

[3] R.S.Timsit: presented at 1988 Int. Conf. Thirty Fourth Meeting of the IEEE Holm, NW.

[4] Q.Y.Yuan: in 2007 Proc. INTERMAG Conf., p. 11

[5] Y. Y. Lau, W. Tang: Journal of Applied Physics, Vol(2009), p. 123

[6] R. S. Timsit: Proceedings of the Thirty Fourth Meeting of the IEEE Holm Conference, p. 26, Sep, 1988

[7] T. S. Narayanan, Y. W. Park and K. Y. Lee: Industrial Lubrication and Tribology, Vol. 60(2008), p.233.

[8] P. G. Luo, L. J. Xu and J. G. Zhang: presented at 2000 Int. Conf. Forty-Sixth IEEE Holm, Langdon.

[9] B. H. Chudnovsky:presented at 2005 Int. Conf. Fifty-First IEEE Holm, Chicago.

[10]M. Runde, E. Hodne, B. Totdal: presented at 1989 Int. Conf. Thirty Fifth Meeting of the IEEE Holm, Chicago.

[11]R. Holm, E. Holm and B. P. Williamson: Springer, Vol. 06(2010), p.11

[12]J. Aronstein, T. K. Hare: Components and Packaging Technologies, Vol. 28(2005), p.701. 